王常顺, 吕汪汪,孙建平,周阳,姜丽丽,李博文, 阿旺,张苏人,夏露,王奇,斯确多吉,贺金生,汪诗平.高寒植物叶片性状对模拟降水变化的响应. 生态学报,2021,41(24): 9760-9772.

Wang C S, Lü W W, Sun J P, Zhou Y, Jiang L L, Li B W, A W, Zhang S R, Xia L, Wang Q, Si Que Duo Ji, He J S, Wang S P. Responses of plant leaf traits to simulated rainfall changes in alpine region. Acta Ecologica Sinica, 2021,41(24):9760-9772.

\title{
高寒植物叶片性状对模拟降水变化的响应
}

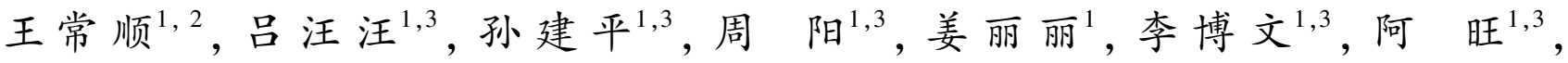
张苏人 ${ }^{1,3}$,夏 露 $^{4}$, 王 奇 ${ }^{1}$, 斯确多吉 ${ }^{1,5}$, 贺金生 ${ }^{6}$, 汪诗平 ${ }^{1,5, *}$

1 中国科学院青藏高原研究所, 北京 100101

2 呼伦贝尔学院, 呼伦贝尔 021008

3 中国科学院大学, 北京 100094

4 西藏大学理学院, 拉萨 850000

5 中国科学院青藏高原地球科学卓越中心, 北京 100101

6 北京大学城市与环境学院, 北京 100080

摘要: 降水变化对高寒草甸生态系统产生了显著影响,植物叶片性状特别是叶脉特征对降水变化非常敏感, 然而高寒植物叶片 性状特征如何响应降水变化还知之较少。采用集雨棚模拟增减 50\%降水的条件, 以高寒草甸 8 种主要植物叶片为研究对象,研 究了降水变化对叶片的叶脉率、叶脉密度、叶片大小、比叶质量、叶片总有机碳含量、叶片全氮含量、叶片碳同位素相对含量和碳 氮比等叶片性状的影响。发现增水显著增加了植物的叶片大小、稳定碳同位素千分值、总有机碳含量、全氮含量, 但显著降低了 叶脉密度; 而减水显著降低了叶片大小、稳定碳同位素千分值。植物叶片性状各指标对降水变化的响应存在协同变化和相互制 约。不同水分生态类型的植物对降水变化的响应存在差异, 中生植物通过增加叶片大小和减少叶脉密度积极应对降水的增加, 矮生嵩草的叶片大小分别增加了 $200.3 \%$, 叶脉密度减小了 $17.5 \%$, 而旱中生植物通过减少叶片大小和增加叶脉密度应对降水 的减少,垂穗披碱草和异针茅的叶片大小分别减少 $54.9 \%$ 和 $30.7 \%$, 其叶脉密度分别增加 $25 \%$ 和 $22.4 \%$ 。羽状叶脉植物增加叶 脉密度和稳定碳同位素千分值以适应增水条件,花苩宿、异叶米口袋的叶脉密度的增加了 $7.8 \%$ 和 $4.0 \%$, 稳定碳同位素千分值 增加 $2.5 \%$ 和 $3.3 \%$, 但增水条件下平行叶脉植物的叶脉密度不变或降低和稳定碳同位素千分值保持不变; 减水增加了平行叶脉 植物叶脉密度并减低了稳定碳同位素千分值, 异针茅的叶脉密度增加了 $22.4 \%$, 稳定碳同位素千分值减小 $2.9 \%$, 而对羽状叶脉 植物的叶脉密度和稳定碳同位素千分值减少或不变。植物叶片性状对增水的敏感性显著大于对减水的敏感性,增水的效应约 为减水的 2 倍; 叶片大小的敏感性显著大于其它叶片性状,约为其它叶片性状的 10 倍。因此,植物在应对短期降水变化时,植 物形态可塑性的作用凸显, 放大或缩小叶片大小是植物应对降水变化的最有效的途径, 但是不同水分生态类型和叶脉类型植物 可塑性的方向存在显著差异。

关键词:气候变化; 高寒草甸; 模拟降水;植物叶片性状; 叶脉特征;协同响应

\section{Responses of plant leaf traits to simulated rainfall changes in alpine region}

WANG Changshun ${ }^{1,2}$, LÜ Wangwang ${ }^{1,3}$, SUN Jianping ${ }^{1,3}$, ZHOU Yang ${ }^{1,3}$, JIANG Lili ${ }^{1}$, LI Bowen ${ }^{1,3}$, A Wang ${ }^{1,3}$, ZHANG Suren ${ }^{1,3}$, XIA Lu ${ }^{4}$, WANG Qi ${ }^{1}$, SI Que Duo Ji ${ }^{1,5}$, HE Jinsheng ${ }^{6}$, WANG Shiping ${ }^{1,5, *}$

1 Institute of Tibetan Plateau Research, Chinese Academy of Sciences, Beijing 100101, China

2 Hulunbuir College, Hulunbuir 021008, China

3 University of Chinese Academy of Sciences, Beijing 100094, China

基金项目: 国家自然科学基金面上项目 (31272488); 国家自然科学基金重点项目 (41230750)

收稿日期: 2020-12-25; 网络出版日期:2021-08-04

* 通讯作者 Corresponding author.E-mail: wangsp@ itpcas.ac.cn 
4 College of Science, Tibet University, Lhasa 850000, China

5 Center of Excellence for Earth Sciences on the Tibetan Plateau, Chinese Academy of Sciences, Beijing 100101, China

6 School of Urban and Environmental Sciences, Peking University, Beijing 100080, China

Abstract : Variations in precipitation had a significant impact on the alpine meadow ecosystem. Among plant leaf traits, leaf vein properties are very sensitive to the changed precipitation. However, how the leaf traits of alpine plants respond to precipitation variations was still little known. The precipitation in the study area was artificially regulated increase and decrease of rain using rainwater shed, and the responses of leaf properties from 8 main plants in the alpine meadow were systematically analyzed under 50\%, 100\%, and 150\% rainfall treatments. This study investigated the effects of altered precipitation on the vein mass per leaf mass (VMM), vein length per area (VLA), leaf size ( LS), leaf mass per area ( LMA), total organic carbon concentration ( TOC), total nitrogen concentration (TN), carbon isotope discrimination $\left(\delta^{13}\right.$ C) and the ratio of carbon to nitrogen $(\mathrm{C} / \mathrm{N})$. The results indicated that water increasing significantly enhanced $\mathrm{LS}, \delta^{13} \mathrm{C}$, TOC, and TN, but significantly reduced for VLA. While water decreasing significantly reduced the LS and $\delta^{13} \mathrm{C}$. There were synergistic changes and mutual constraints in the response of plant leaf traits to precipitation alteration. Plants belonging to different water ecological groups had different responses to precipitation alteration. Mesophytes actively responded to the increase in precipitation by increasing LS and reducing VLA. The LS of Kobresia humilis was increased by $200.3 \%$, and the VLA was reduced by $17.5 \%$. While dry mesophytes responded to the decrease in precipitation by reducing LS and increasing VLA. The LS of Elymus nutans and Stipa purpure decreased by $54.9 \%$ and $30.7 \%$, and their VLA increased by $25 \%$ and $22.4 \%$, respectively. Pinnate vein plants could enhance VLA and $\delta^{13} \mathrm{C}$ in order to adapt to water increasing condition. The VLA of Medicago ruthenica and Gueldenstaedtia diversifolia increased by $7.8 \%$ and $4.0 \%$, and the $\delta^{13} \mathrm{C}$ increased by $2.5 \%$ and 3.3\%. While for parallel vein plants, they remained unchang or decrease. Water reducing enhanced VLA of parallel veins plant and reduced $\delta^{13} \mathrm{C}$, while had no impact on pinnate vein plants. The VLA of $S$. purpure increased by $22.4 \%$ and the $\delta^{13} \mathrm{C}$ decreased by $2.9 \%$. The plant leaf traits were much more significantly sensible to water increasing than water reducing, and the effect of water increasing was about 2 times than that of water reduction. LS was the most significantly sensitive among all leaf traits, about 10 times that of other leaf traits. Therefore, plant morphology plasticity played a prominent role when responding to short-term precipitation variation. Enlarging or reducing leaf size was the most effective way to respond to precipitation alteration. However, plant plasticity was significantly different among the water ecological types and leaf vein types.

Key Words : climate change, alpine meadow, simulated precipitation, plant leaf traits, vein traits, synergistic response

植物叶片性状是植物与环境相互作用的结果, 能够客观反映植物对外部环境变化的响应和适应 ${ }^{[1-2]}$, 因 此, 研究植物的这些性状的环境驱动机制为预测未来从器官到个体的响应提供可靠的研究手段 ${ }^{3-5]}$ 。在过去 几十年中, 青藏高原大多数地区降水增加, 特别是在高原东部和南部 ${ }^{[6-7]}$ 。由于高寒草甸区域属于半干旱半 湿润地区 ${ }^{8]}$ 。因此,未来降水格局的变化可能对高寒草甸生态系统带来显著地影响,这样的影响驱动着青藏 高原草甸群落物种叶片性状的变化 ${ }^{[9]}$ 。然而现有对于高寒草甸对气候变化的响应的研究多从生态系统 ${ }^{[10]}$ 、 群落 $^{[11]}$ 、种群 ${ }^{[8]}$ 等层面开展。目前的研究仍然不能确切的回答高寒区植物叶片性状对降水变化的响应过程 和机制。

目前对于植物叶片性状耐旱特性的研究较为丰富 ${ }^{[12-13]}$, 但是植物叶片性状对干旱的适应机制与植物对

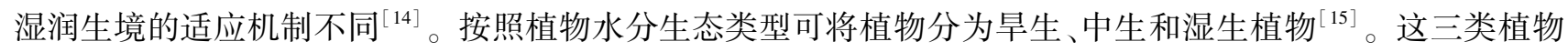
对干旱的抗性依次减弱, 在水分充裕的环境中其竞争力依次增强 ${ }^{[16]}$ 。而在相对湿润的高寒草甸, 以中生植物 (如矮生嵪草 (Kobresia humilis)、高山嵩草 (Kobresia pygmaea)) 为建群种, 同时伴生有旱生的禾本科植物 (如 紫花针茅 (Stipa purpurea $)^{[17]}$ 。由于旱生植物通常具有特殊的叶片抗旱结构 ${ }^{[18]}$, 所以旱生植物更能适应干旱 
环境; 同时由于湿生植物无需负担适应干旱的叶片结构 ${ }^{[19]}$, 所以在湿润环境中的竞争力更高。因此,不同水 分生态类型的植物叶片性状对于降水增减变化的响应可能存在差异。然而此类的研究还很缺乏,尤其缺乏基 于站点的模拟气候变化研究, 难以从器官的响应的角度提供可靠的结论 ${ }^{[20]}$ 。

叶脉是植物进行水分输送的通道, 叶脉性状可以影响水分的传输效率, 进而影响植物的光合作用 ${ }^{[2] 。}$ 有 研究表明植物通过增加其单位面积上的叶脉长度来提高对干旱适应能力 ${ }^{[21]}$ 。虽然不同叶脉类型植物的叶脉 密度比较接近 ${ }^{[22]}$, 但是有研究表明平行叶脉植物的叶脉投人远高于羽状叶脉的植物 ${ }^{[23-24]}$, 并且不同叶脉类型 植物对气候的适应策略存在差异 ${ }^{[25]}$ 。然而不同叶脉类型的植物叶片性状对降水变化的响应是否存在差异仍 然不明确。

植物叶片性状的变异性和可塑性是最大的, 故以植物叶片性状为研究指标, 更能反映出植物对气候变化 的响应过程和机制 ${ }^{[26]}$, 基于以上认识, 本研究拟讨论高寒草甸不同水分生态类型和不同叶脉类型植物叶片性 状对模拟降水变化的响应。

\section{1 实验样地}

模拟降水实验样地设立在中国科学院海北高寒草甸生态系统定位研究站 (简称海北站) 的综合观测场 $\left(37^{\circ} 29^{\prime} \mathrm{N}, 101^{\circ} 12^{\prime} \mathrm{E}\right)$ 。该站位于青藏高原的东北隅, 地处祁连山东段北支冷龙岭南麓, 大通河河谷的西北 部, 站区以山间滩地和丘陵低山为主, 滩地海拔 $3200 \mathrm{~m}$ 。该地区气候为高原大陆性气候, 以适应寒冷湿中生 的多年生草本植物群落为主要植被类型 ${ }^{[27]}$ 。矮生嵩草草甸为站区主要植被类型, 由多年生草本植物组成, 优 势种和建群种为矮生嵩草。矮生嵩草草甸植物生长低矮, 但群落总覆盖度较高, 植物种类组成较多, 但群落结 构为单层结构, 其植物群落以矮生嵩草、麻花艽 (Gentiana straminea) 、异针茅 ( Stipa aliena)、花苜宿 (Medicago ruthenica)、垂穗披碱草 (Elymus nutans $)$ 等为优势种 ${ }^{[27]}$ 。土壤为草毡寒冻雏形土 ${ }^{[27]}$ 。

\section{2 研究方法}

\section{1 实验设计}

减水 $50 \%$ 的处理采用集雨棚来模拟减水, 4 个透明的聚碳酸树脂通道(面积占整个小区面积的 $50 \%$ ) 以 $15^{\circ}$ 固定在距地表 $160 \mathrm{~cm}$ 处,截留的雨水通过管道流人白色聚乙烯塑料雨水采集器内。每次及时将减水处理 小区采集器的雨水添加到增水的小区, 以确保达到增水 $50 \%$ 的目的。为减少或消除辐射器遮荫或其它因素 造成的实验误差, 在相应对照小区上方均设 4 个虚拟透明的聚碳酸树脂通道, 通道的底部开口, 以实现降水不 减少。另外在每个小区四周埋人铁皮以减少地表径流的影响。每个实验小区面积为 $1.5 \mathrm{~m} \times 1.5 \mathrm{~m}$ 。实验起 始于 2011 年。实验重复 3 个。

\section{2 样品采集与测量}

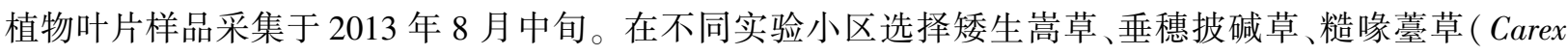
scabrirostris)、花苜宿、麻花艽、异叶米口袋 (Gueldenstaedtia diversifolia) 、雪白委陵菜 ( Potentilla nivea)、异针茅 8 种植物, 每种植物选取 3-5 株, 采集自植物顶端第二片或第三片成熟叶片, 分别测量叶片的叶脉率、叶脉密 度、叶片大小、比叶质量、叶片总有机碳含量、叶片全氮含量、叶片碳同位素相对含量和碳氮比等指标。

叶脉率(Vein mass per leaf mass, VMM) 测量选取自顶端第二或三片成熟的植物叶片 5 片, 分别在 $60^{\circ} \mathrm{C}$ 下 烘干并称重。烘干样品在 5\% 的氢氧化钠溶液中煮沸 1-3 min, 直到非常柔软, 但是不能散乱, 然后在放大镜 下用镊子小心分离叶脉和叶肉。分离出的叶脉在 $60^{\circ} \mathrm{C}$ 下烘干并称重, 所用天平为 XP6 (Balance Mettlertoledo, Bern, Switzerland), 精度为百万分之一克。计算叶脉占叶质量的百分比 $(\%)$ 。尽管细叶脉占叶脉总长 度的 $80 \%$, 但是其质量只占叶脉总质量的 $4 \%{ }^{[28]}$, 所以忽略了在叶脉分离过程中丢失的部分细叶脉的质量。

叶脉密度 (Vein length per area, VLA) 测量使用Ueno 等采用的方法 ${ }^{[29]}$, 单位为 $\mathrm{mm} / \mathrm{mm}^{2}$ 。叶片大小 ( Leaf size, LS) 测量使用 Luo 等采用的方法 ${ }^{[30]}$, 单位为 $\mathrm{mm}^{2}$ 。比叶质量 (Leaf mass per area, LMA) 采用 Niklas 等采 
用的方法 ${ }^{[31]}$, 单位为 $\mathrm{g} / \mathrm{m}^{2}$ 。

叶片总有机碳含量 ( Total organic carbon concentration, TOC) ) 和叶片全氮含量 ( Total nitrogen concentration, TN) 使用碳氮分析仪测量 (2400 CHNS/O, Perkin-Elmer, USA), 单位为\%。叶片稳定碳同位素 千分值 (Carbon isotope discrimination, $\delta^{13} \mathrm{C}$ ) 采用冯秋红等使用方法 ${ }^{[32]}$,单位为\%o。

为比较不同量纲的叶片性状的变化情况, 本文创建“降水敏感性”概念, 即单位降水幅度下, 叶片性状的 变化率。每种叶片性状的降水敏感性计算公式如下:

降水敏感性 $(\% / \%)=($ 处理-对照 $) /$ 对照/降水变幅 $\times 100 \%)$

\section{3 数据分析}

采用一般线性模型 (GLM) 中的双因素方差分析 (Two-way ANOVA) 分别检验处理(增水和减水)、物种及 其互作效应对某一叶片性状( 叶脉率、叶脉密度、叶片大小、比叶质量、叶片总有机碳含量、叶片全氮含量、叶 片碳同位素相对含量和碳氮比) 的显著性 ${ }^{[33]}$, 并使用多重比较 (Duncan test) 检验不同物种某一叶片性状的 差异。

采用一般线性模型 (GLM) 中的三因素方差分析( three-way ANOVA) 检验水分、物种和性状及其互作效应 对叶片性状水分敏感性的显著性 ${ }^{[33]}$, 并使用多重比较 (Duncan test) 检验不同物种某一叶片性状和物种的差 异。统计分析时用敏感性的绝对值进行比较分析。

采用 Pearson 法计算所有物种和单一物种叶片性状之间的相关关系。分析软件使用 SPSS 18.0,所有显著 性水平都是 $P<0.05$ 。

\section{3 结果与分析}

3.1 叶片性状对降水变化的响应

总体而言, 降水变化对植物叶片大小、叶脉密度、稳定碳同位素千分值、总有机碳含量和全氮含量具有显 著作用; 对叶脉率、比叶质量和碳氮比无显著作用 (表 1)。说明高寒区的降水变化对植物叶片性状具有显著 影响,但是不同性状的具体表现存在差异。不同物种之间的叶片性状均有显著差异 (表 1)。除了全氮含量和 碳氮比以外, 不同物种的同一性状对降水变化的响应均具有显著差异 (表 1)。说明降水变化对叶片性状的影 响因物种而异,叶片性状对降水变化的响应并没有普适的规律。

具体而言, 增水显著增加了植物的叶片大小、稳定碳同位素千分值、总有机碳含量、全氮含量, 分别由 $(516.16 \pm 567.68) \mathrm{mm}^{2} 、(-28.4 \pm 1.33) \%$ 、 $(43.96 \pm 3.67) \% 、(2.4 \pm 0.25) \%$ 增加到 $(645.81 \pm 551.03) \mathrm{mm}^{2} 、$ $(-28.15 \pm 1.07) \%$ 、 $(45.95 \pm 3.38) \% 、(2.57 \pm 0.29) \%$; 显著降低了叶脉密度, 由 $(6.03 \pm 0.75)$ 降低到 $(5.83 \pm 0.95)$ $\mathrm{mm} / \mathrm{mm}^{2}$; 对叶脉率、碳氮比和比叶质量无显著影响, 平均为 $(26.06 \pm 17.82) \% 、(18.46 \pm 2.16)$ 和 $(98.91 \pm 39.17)$ $\mathrm{g} / \mathrm{m}^{2}$ (表 2)。减水显著降低了叶片大小、稳定碳同位素千分值, 降低到 $(430.06 \pm 568.65) \mathrm{mm}^{2}$ 和 $(-28.83 \pm$ 1.09 )\% ; 对总有机碳含量、全氮含量、叶脉密度、叶脉率、碳氮比和比叶质量无显著影响 (表 2)。植物的叶片 大小、稳定碳同位素千分值可以很好的指示降水的增减变化,而叶脉率、碳氮比和比叶质量对降水变化的响应 不明显,部分叶片性状对降水增减变化不对称。

就具体物种同一性状而言,增水显著增加了矮生嵩草、花苜宿、异叶米口袋、雪白委陵菜的叶片大小,但对

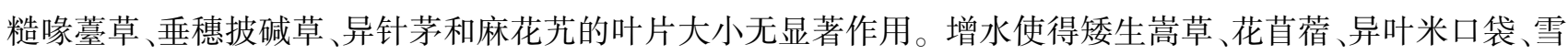
白委陵菜的叶片大小分别增加了 $200.3 \%$ 、258.6\%、289.4\% 和 53.3\%(图 1)。减水显著减小了垂穗披碱草和异 针茅的叶片大小, 但对矮生嵩草、粘嚎臺草、花苜宿、异叶米口袋、麻花艽、雪白委陵菜的叶片大小无显著作用。 减水使得垂穗披碱草和异针茅的叶片大小分别减少 54.9\%和 30.7\%(图 1)。降水的增减变化,对不同物种叶 片大小的作用存在差异, 部分物种对增加降水的响应更为积极, 表现为叶片大小的增加; 而部分物种对降水减 少的响应更为积极, 表现为叶片大小的减少。 
表 1 降水和物种对叶片性状双因素方差分析表

Table 1 Two-way ANOVA of leaf traits under adding and reducing water between different species

\begin{tabular}{|c|c|c|c|c|}
\hline $\begin{array}{l}\text { 误差源 } \\
\text { Error source }\end{array}$ & $\begin{array}{l}\text { 叶片性状 } \\
\text { Leaf traits }\end{array}$ & $\begin{array}{l}\text { 单位 } \\
\text { Unit }\end{array}$ & $d f$ & $P$ \\
\hline 降水 & 叶片大小 ( LS) & $\mathrm{mm}^{2}$ & 2 & $<0.001$ \\
\hline \multirow[t]{7}{*}{ Precipitation } & 叶脉密度 (VLA) & $\mathrm{mm} / \mathrm{mm}^{2}$ & 2 & 0.01 \\
\hline & 叶脉率( VMM) & $\%$ & 2 & 0.89 \\
\hline & 比叶质量 ( LMA) & $\mathrm{g} / \mathrm{m}^{2}$ & 2 & 0.27 \\
\hline & 稳定碳同位素千分值 $\left(\delta^{13} \mathrm{C}\right)$ & $\%$ & 2 & $<0.001$ \\
\hline & 总有机碳含量( TOC) & $\%$ & 2 & $<0.001$ \\
\hline & 全氮含量( TN) & $\%$ & 2 & $<0.001$ \\
\hline & 碳氮比(C/N) & & 2 & 0.10 \\
\hline 物种 & 叶片大小 ( LS) & $\mathrm{mm}^{2}$ & 7 & $<0.001$ \\
\hline \multirow[t]{7}{*}{ Species } & 叶脉密度 (VLA) & $\mathrm{mm} / \mathrm{mm}^{2}$ & 7 & $<0.001$ \\
\hline & 叶脉率( VMM) & $\%$ & 7 & $<0.001$ \\
\hline & 比叶质量( LMA) & $\mathrm{g} / \mathrm{m}^{2}$ & 7 & $<0.001$ \\
\hline & 稳定碳同位素千分值 $\left(\delta^{13} \mathrm{C}\right)$ & $\%$ & 7 & $<0.001$ \\
\hline & 总有机碳含量( TOC) & $\%$ & 7 & $<0.001$ \\
\hline & 全氮含量( TN) & $\%$ & 7 & $<0.001$ \\
\hline & 碳氮比(C/N) & & 7 & $<0.001$ \\
\hline 降水×物种 & 叶片大小 ( LS) & $\mathrm{mm}^{2}$ & 14 & $<0.001$ \\
\hline \multirow[t]{7}{*}{ Precipitation $\times$ Species } & 叶脉密度 (VLA) & $\mathrm{mm} / \mathrm{mm}^{2}$ & 14 & $<0.001$ \\
\hline & 叶脉率( VMM) & $\%$ & 14 & $<0.001$ \\
\hline & 比叶质量 ( LMA) & $\mathrm{g} / \mathrm{m}^{2}$ & 14 & 0.02 \\
\hline & 稳定碳同位素千分值 $\left(\delta^{13} \mathrm{C}\right)$ & $\%$ & 14 & $<0.001$ \\
\hline & 总有机碳含量( TOC) & $\%$ & 14 & 0.049 \\
\hline & 全氮含量( TN) & $\%$ & 14 & 0.60 \\
\hline & 碳氮比(C/N) & & 14 & 0.05 \\
\hline
\end{tabular}

LS: 叶片大小 Leaf size; VLA: 叶脉密度 Vein length per area;VMM: 叶脉率 Vein mass per leaf mass; LMA: 比叶质量 Leaf mass per area; $\delta^{13} \mathrm{C}$ : 稳 定碳同位素千分值 Carbon isotope discrimination; TOC: 总有机碳含量 Total organic carbon concentration; TN: 全氮含量 Total nitrogen concentration; $\mathrm{C} / \mathrm{N}$ :碳氮比 Carbon to nitrogen ratio

\section{表 2 叶片性状对降水变化的响应差异}

Table 2 Differences of leaf traits in response to precipitation changes

\begin{tabular}{|c|c|c|c|c|}
\hline & $\begin{array}{l}\text { 叶片大小 } \\
\mathrm{LS} / \mathrm{mm}^{2}\end{array}$ & $\begin{array}{c}\text { 叶脉密度 } \\
\mathrm{VLA} /\left(\mathrm{mm} / \mathrm{mm}^{2}\right)\end{array}$ & $\begin{array}{c}\text { 叶脉率 } \\
\mathrm{VMM} / \%\end{array}$ & $\begin{array}{c}\text { 比叶质量 } \\
\mathrm{LMA} /\left(\mathrm{g} / \mathrm{m}^{2}\right)\end{array}$ \\
\hline 对照 Control & $516.16 \pm 567.68 \mathrm{~b}$ & $6.03 \pm 0.75 \mathrm{a}$ & $26.06 \pm 17.82 \mathrm{a}$ & $98.91 \pm 39.17 \mathrm{a}$ \\
\hline 增水 Adding water & $645.81 \pm 551.03 \mathrm{a}$ & $5.83 \pm 0.95 b$ & $25.50 \pm 19.7 \mathrm{a}$ & $99.02 \pm 38.78 \mathrm{a}$ \\
\hline \multirow[t]{2}{*}{ 减水 Reducing water } & $430.06 \pm 568.65 \mathrm{c}$ & $6.11 \pm 0.87 \mathrm{a}$ & $25.50 \pm 19.33 \mathrm{a}$ & $96.85 \pm 40.06 \mathrm{a}$ \\
\hline & $\begin{array}{c}\text { 稳定碳同位素千分值 } \\
\delta^{13} \mathrm{C} / \% \%\end{array}$ & $\begin{array}{c}\text { 总有机碳含量 } \\
\text { TOC/\% }\end{array}$ & $\begin{array}{c}\text { 全氮含量 } \\
\text { TN/\% }\end{array}$ & $\begin{array}{l}\text { 碳氮比 } \\
\mathrm{C} / \mathrm{N}\end{array}$ \\
\hline 对照 Control & $-28.40 \pm 1.33 b$ & $43.96 \pm 3.67 \mathrm{~b}$ & $2.40 \pm 0.25 b$ & $18.46 \pm 2.16 \mathrm{ab}$ \\
\hline 增水 Adding water & $-28.15 \pm 1.07 \mathrm{a}$ & $45.95 \pm 3.38 \mathrm{a}$ & $2.57 \pm 0.29 \mathrm{a}$ & $18.03 \pm 2.15 b$ \\
\hline 减水 Reducing water & $-28.83 \pm 1.09 c$ & $41.10 \pm 4 b$ & $2.36 \pm 0.27 b$ & $18.84 \pm 2.44 \mathrm{a}$ \\
\hline
\end{tabular}

不同字母代表具有显著差异 $(P<0.05)$

增水显著增加花苜宿、异叶米口袋的叶脉密度, 其叶脉密度分别增加了 $7.8 \% 、 4.0 \%$; 增水使得矮生嵩草 的叶脉密度显著减小了 $17.5 \%$ (图 2)。增水对雪白委陵菜、糙嚎臺草、垂穗披碱草、麻花艽和异针茅的叶脉密 度无显著作用。减水显著增加了垂穗披碱草和异针茅的叶脉密度,其叶脉密度分别增加 $25 \%$ 和 $22.4 \%$; 减水 使得花苜宿、异叶米口袋、麻花艽和雪白委陵菜的叶脉密度分别显著减少 5.5\%、2.7\%、4.6\%和 19.7\%(图 2)。 减水对矮生嵩草和粘嚎臺草的叶脉密度无显著作用。降水的增减变化,对不同物种叶脉密度的作用存在差 


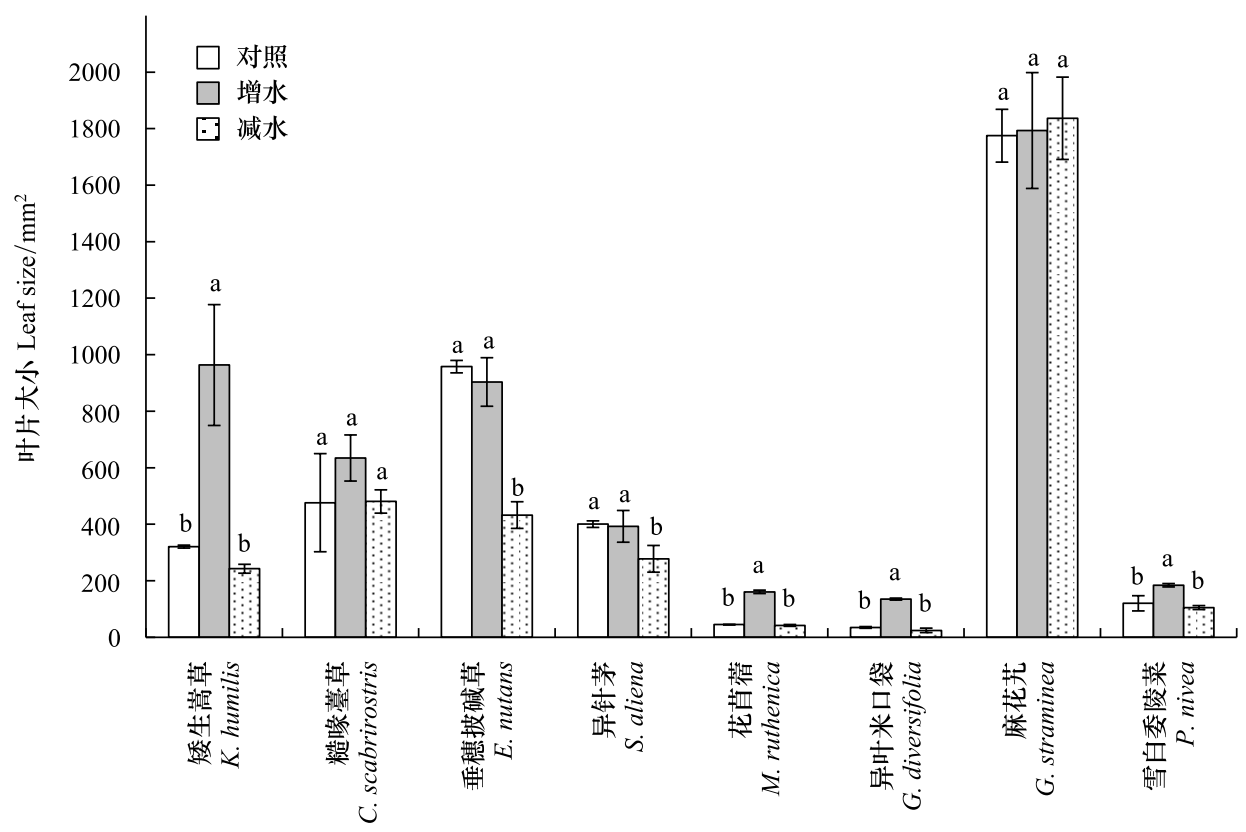

图 1 不同植物叶片大小对增水和减水的响应

Fig.1 The response of different plant's LS to adding and reducing water, the different words means significant difference 不同字母代表具有显著差异 $(P<0.05)$

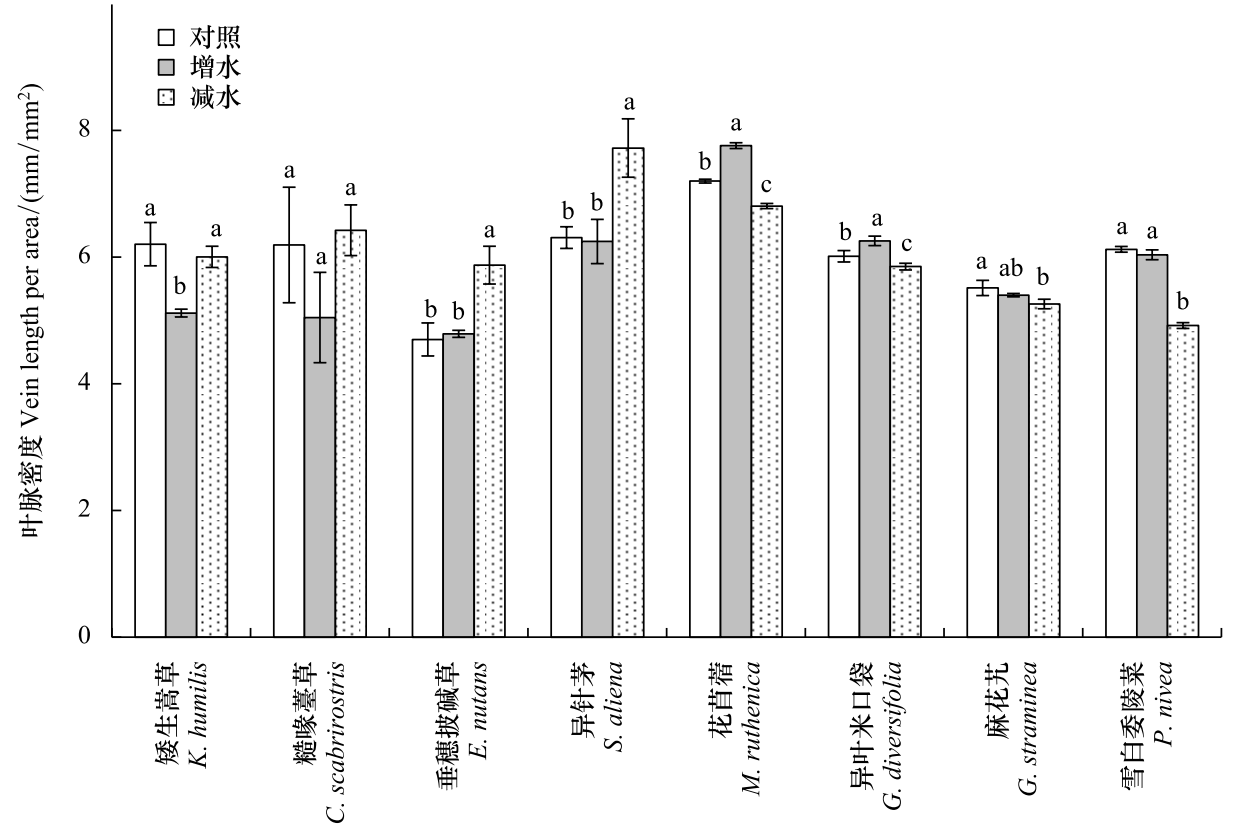

图 2 不同植物叶脉密度对增水和减水的响应

Fig.2 The response of different plant's VLA to adding and reducing water, the different words means significant difference between contral and warming

不同字母代表具有显著差异 $(P<0.05)$

异,甚至完全相反,尤其是不同叶脉类型的植物的响应表现出相反的变化方向,说明不同植物应对降水变化 时,其叶脉密度的变化策略存在较大差异,并与植物叶脉类型相关。

增水使得垂穗披碱草的叶脉率显著增加 $23.6 \%$; 增水显著降低异叶米口袋叶脉率,其叶脉率降低 $74.4 \%$ 。

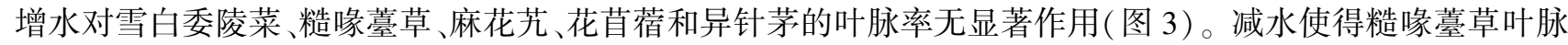


率显著增加 $29.4 \%$ 。减水对垂穗披碱草、异针茅、矮生嵩草、麻花艽、花苜宿、异叶米口袋、雪白委陵菜的叶脉 率无显著作用(图 3)。与叶脉密度的变化相类似,当降水发生增减变化时,不同叶脉类型植物的叶脉率的变 化方向表现出较大不同,甚至完全相反。

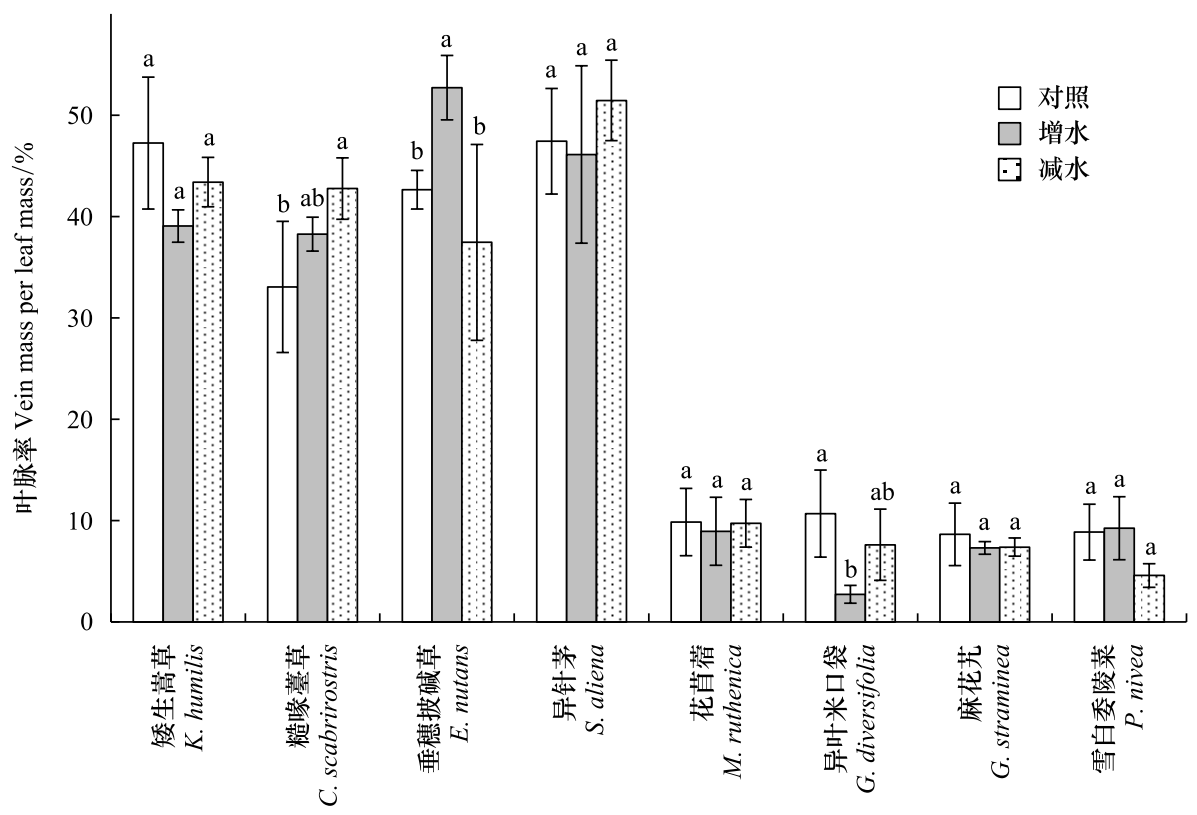

图 3 不同植物叶脉率对增水和减水的响应

Fig.3 The response of different plant's VMM to adding and reducing water, the different words means significant difference between contral and warming

不同字母代表具有显著差异 $(P<0.05)$

增水使得花苜宿、异叶米口袋、雪白委陵菜的稳定碳同位素千分值分别显著增加 $2.5 \%$ 、3.3\% 和 $5.4 \%$ (图 4)。增水对矮生嵩草、糙喙薹草、垂穗披碱草、麻花艽和异针茅的稳定碳同位素千分值无显著作用。减水

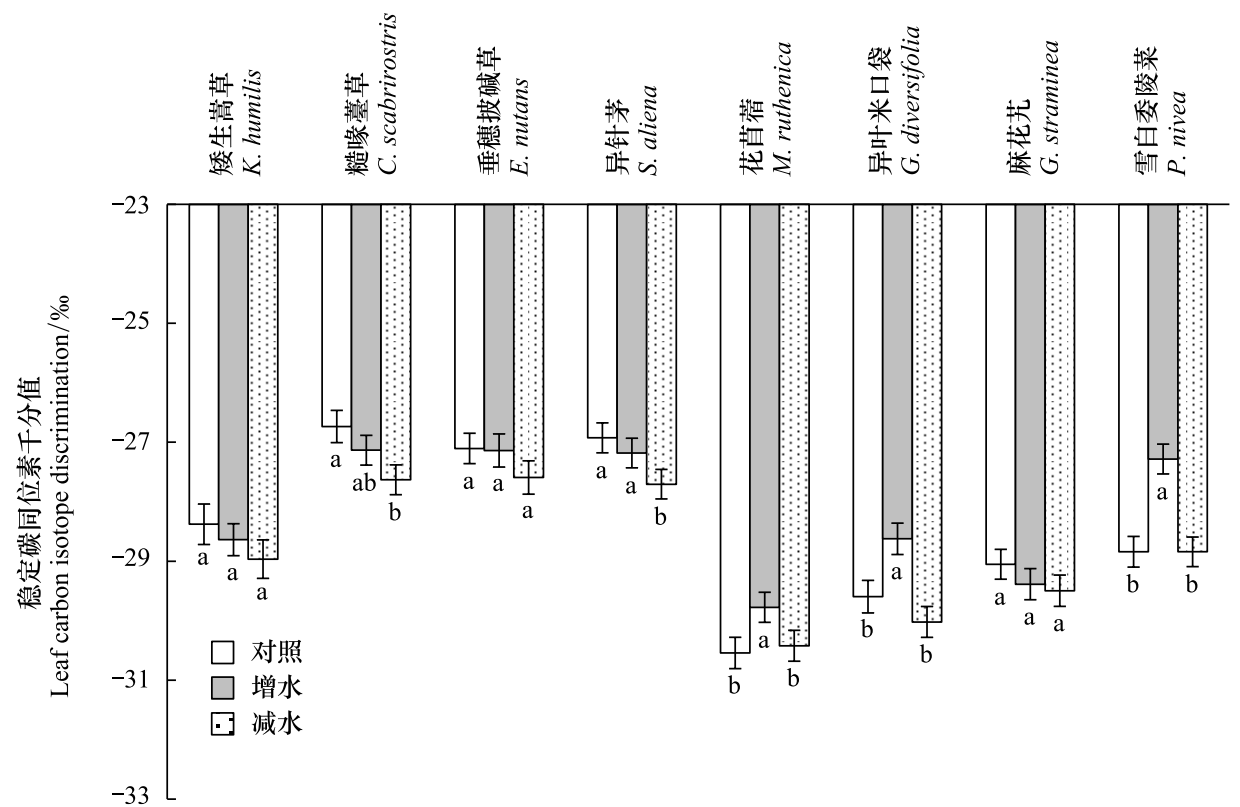

图 4 不同植物稳定碳同位素千分值对增水和减水的响应

Fig.4 The response of different plant's $\delta^{13} \mathrm{C}$ to adding and reducing water, the different words means significant difference between contral and warming

不同字母代表具有显著差异 $(P<0.05)$ 
使得粘嗄荎草和异针茅的稳定碳同位素千分值显著减小 $3.3 \%$ 和 $2.9 \%$ (图 4)。减水对垂穗披碱草、矮生嵩 草、花苜宿、异叶米口袋、麻花艽、雪白委陵菜的稳定碳同位素千分值无显著作用。降水的增减变化,对不同物 种稳定碳同位素千分值的作用存在差异, 部分羽状叶脉植物对增加降水的响应更为积极, 表现为稳定碳同位 素千分值的增加; 而部分平行叶脉植物对降水减少的响应更为积极,表现为稳定碳同位素千分值的减少。

3.2 叶片性状降水敏感性对降水变化的响应

降水变化、物种和叶片性状及其互作效应显著影响叶片性状响应的敏感性(表 3)。植物叶片性状对增水 的敏感性显著大于减水,约为减水的 2 倍, 说明本研究所涉及的物种对于降水的增加响应更为积极, 同时对降 水减少具有一定的适应性 (图 5); 叶片大小的敏感性显著大于其它叶片性状,约为其它叶片性状 10 倍, 说明 叶片大小的变化可以很好的指示植物对降水变化的响应 (图 5); 矮生嵩草、花苩宿、异叶米口袋的三种植物的 敏感性较大, 而粘㖨萄草、垂穗披碱草、异针茅、麻花艽和雪白委陵菜敏感性较低(图 5), 说明不同物种叶片性 状对降水变化的响应存在较大差异。

表 3 降水、性状和物种对敏感性三因素方差分析表

Table 3 Three-way ANOVA of sensitivity among different precipitation, species and leaf traits

\begin{tabular}{lcc}
\hline 误差源 Source & $d f$ & $P$ \\
\hline 降水 Precipitation & 1 & $<0.001$ \\
物种 Species & 7 & $<0.001$ \\
性状 Traits & 7 & $<0.001$ \\
物种×降水 Species×Precipitation & 7 & $<0.001$ \\
物种×性状 Species×Traits & 49 & $<0.001$ \\
性状×降水 Traits×Precipitation & 7 & $<0.001$ \\
物种×降水×性状 Species×Precipitation×Traits & 49 & $<0.001$ \\
\hline
\end{tabular}

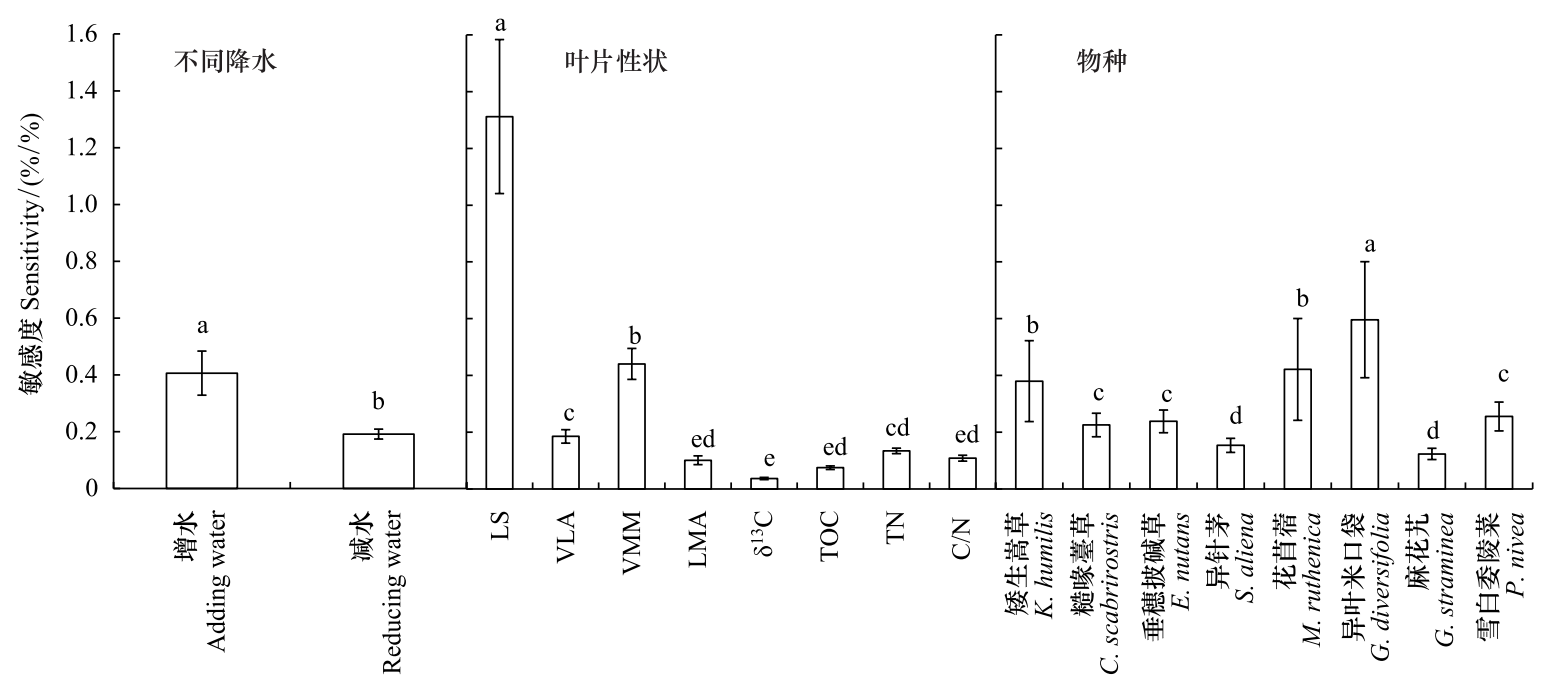

图 5 不同降水、叶片性状和物种之间敏感性的差异

Fig.5 The difference sensitivity of different precipitation, leaf traits and species, different letter means significant different between species 不同字母代表具有显著差异 $(P<0.05)$; LS : 叶片大小 Leaf size; VLA: 叶脉密度 Vein length per area; VMM : 叶脉率 Vein mass per leaf mass; LMA: 比叶质量 Leaf mass per area; $\delta^{13} \mathrm{C}$ : 稳定碳同位素千分值 Carbon isotope discrimination; TOC: 总有机碳含量 Total organic carbon concentration; TN : 全氮含量 Total nitrogen concentration; $\mathrm{C} / \mathrm{N}$ : 碳氮比 Carbon to nitrogen ratio

3.3 叶片性状之间的相互关系

比叶质量与叶脉率、稳定碳同位素千分值与叶脉率和比叶质量、总有机碳含量与叶片大小和比叶质量、总 有机碳含量与稳定碳同位素千分值、全氮含量与叶脉率和稳定碳同位素千分值、全氮含量与总有机碳含量、碳 
氮比与叶片大小和总有机碳含量、碳氮比与比叶质量呈显著的正相关 (表 4); 叶脉密度与叶片大小和碳氮比 与全氮含量呈显著的负相关 (表 4)。说明植物叶片性状并非完全独立, 某一性状的变化可能伴随着其它性状 的变化。叶脉密度有是限制叶片大小变化的重要因素。

表 4 叶片性状之间的 Pearson 相关系数

Table 4 The Pearson correlation coefficient of leaf traits

\begin{tabular}{lccccccc}
\hline & $\begin{array}{c}\text { 叶脉密度 } \\
\text { VLA }\end{array}$ & $\begin{array}{c}\text { 叶脉率 } \\
\text { VMM }\end{array}$ & $\begin{array}{c}\text { 比叶质量 } \\
\text { LMA }\end{array}$ & $\begin{array}{c}\text { 稳定碳同位素 } \\
\text { 千分值 } \delta^{13} \mathrm{C}\end{array}$ & $\begin{array}{c}\text { 总有机碳含量 } \\
\text { TOC }\end{array}$ & $\begin{array}{c}\text { 全氮含量 } \\
\text { TN }\end{array}$ & $\begin{array}{c}\text { 碳氮比 } \\
\mathrm{C} / \mathrm{N}\end{array}$ \\
\hline 叶片大小 LS & $-0.477^{*}$ & 0.014 & 0.128 & 0.078 & $0.421^{*}$ & -0.102 & $0.459^{*}$ \\
叶脉密度 VLA & & -0.025 & -0.017 & -0.214 & -0.172 & 0.049 & -0.200 \\
叶脉率 VMM & & & $0.610^{*}$ & $0.709^{*}$ & 0.164 & $0.339^{*}$ & -0.222 \\
比叶质量 LMA & & & & $0.774^{*}$ & $0.572^{*}$ & 0.166 & $0.240^{*}$ \\
稳定碳同位素千分值 $\delta^{13} \mathrm{C}$ & & & & & $0.439^{*}$ & $0.484^{*}$ & -0.160 \\
总有机碳含量 TOC & & & & & & $0.372^{*}$ & $0.368^{*}$ \\
全氮含量 TN & & & & & & & $-0.716^{*}$ \\
\hline
\end{tabular}

*代表显著相关 $(P<0.05)$

\section{4 讨论}

\section{1 不同叶片性状对降水变化的协同响应及其相互制约}

叶片大小直接影响着植物对光的截取和碳获取的能力 ${ }^{[34]}$ 。本研究的结果表明: 增水显著增加了大部分 植物的叶片大小; 而减水则显著减少了大部分植物的叶片大小 (表 2), 这与已往的研究结果一致 ${ }^{[35-37]}$ 。群落 的平均叶片大小与降水直接相关 ${ }^{[35]}$ 。越干旱, 同一物种叶片越小 ${ }^{[36-37]}$ 。如马蹄香( Primula siebodii) 的叶片 大小在水分亏缺时比湿润时更小 ${ }^{[38]}$ 。澳大利亚东南部的多年生植物的叶宽与降水量呈正相关 ${ }^{[39]}$ 。这主要 是因为大叶需要更多的水分蒸腾来降低温度; 而水分供给不足时, 植物通过减少叶片大小来减少水分的消耗, 同时防止叶温过高。叶片大小减少可以改变叶片的气体传导率, 从而降低高蒸发带来的伤害 ${ }^{[40]}$, 所以降水的 变化直接影响着叶片大小, 进而影响植物的生产力。植物形态要比植物功能更具有可塑性 ${ }^{[17,41]}$ 。叶片大小 可以很好的指示环境中水分资源的盈亏。

叶脉对光合组织起到支撑和传输的作用 ${ }^{[24]}$, 因此叶脉密度的变化表征了光合组织对支撑和传输需求的 变化。本研究的结果表明:总体上减水对叶脉密度无显著作用,而增水显著降低了叶脉密度 (表 3 )。水分条 件的改善可能对传输组织的需求下降,所以可能是叶脉密度下降的原因; 而叶脉密度的增加有利于抗旱能力 的提高 ${ }^{[42]}$, 可能是减水条件下叶脉密度不变的原因。

水分控制实验已开展 3 年,植物叶片发生显著变化。总体上,本研究监测的 8 种叶片性状中有 5 种对降 水变化的响应显著。随着叶片大小的变化,其它叶片性状也有不同程度的改变 (表 1)。叶片性状之间的关系 表明: 叶片大小与总有机碳含量和碳氮比呈显著正相关,而与叶脉密度呈显著负相关 (表 4)。例如: 增水显著 增加了植物的叶片大小, 同时显著增加了总有机碳含量和显著降低了叶脉密度 (表 2)。这表明植物叶片性状 之间具有协同变化。不同的叶片性状具有不同的功能 ${ }^{[43]}$ 。叶片大小的增加可以增加生产力 ${ }^{[34]}$, 而总有机碳 含量的增加是植物趋于保守的投资策略 ${ }^{[4]}$, 同时叶脉密度的降低将减少水分传输的能力, 可能限制光合能力 和生产力的进一步增加 ${ }^{[24]}$ 。这意味着植物在应对降水变化时既有积极的一面也有保守的一面, 不同叶片性 状存在协同响应和相互制约的关系。

4.2 不同水分生态类型植物叶片性状对降水变化的响应

增水显著增加了矮生嵩草、花苩宿、异叶米口袋、雪白委陵菜的叶片大小, 分别增加了叶片大小 (图 1)。 而对粘喙萄草、垂穗披碱草、麻花艽、和异针茅的叶片大小无显著作用。垂穗披碱草和异针茅是属于旱中生植 物, 而其它物种属于中生植物 ${ }^{[15]}$ 。虽然糙鲒莒草叶片大小没有显著变化, 但是增水增加其叶片大小的 $33 \%$ 
（图 1)。显然中生植物比旱中生对湿润条件的适应能力更强。所以增水对中生植物叶片大小的影响较显著, 而对旱中生植物的叶片大小的影响较小。

与增水相反,减水显著减小垂穗披碱草、异针茅的叶片大小 (图 1)。而减水对矮生嵩草、粘㗒薹草、花苜 宿、异叶米口袋、麻花艽、雪白委陵菜的叶片大小无显著作用。减水对旱中生植物叶片大小的影响显著,而对 中生植物的叶片大小作用较小。植物通过改变其叶片性状来响应降水的变化,最大限度地达到其表型与生存 环境相一致而利用或缓冲环境对生长和生殖产生的积极或消极影响 ${ }^{[45]}$ 。所以垂穗披碱草和异针茅旱中生植 物可以通过缩小叶片大小对干旱有更强的抗性，而其它 6 种中生植物通过增加叶片大小来适应湿润环境。

增水显著减小矮生嵩草的叶脉密度,对垂穗披碱草和异针茅的叶脉密度无显著作用(图 2)。虽然增水没

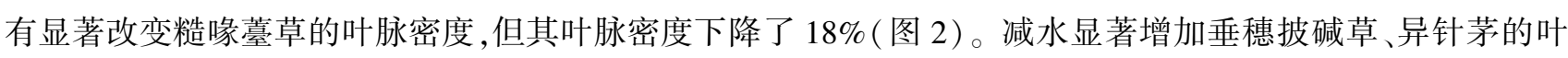

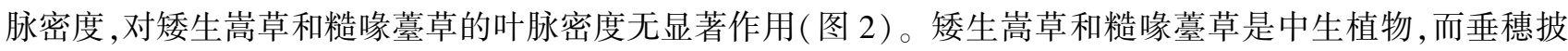
碱草和异针茅是旱中生植物 ${ }^{[15]}$ 。降低叶脉密度可以减少对构建消耗较高的叶脉组织的投人 ${ }^{[46]}$, 进而将有限 的资源用于其他组织。而增加叶脉密度可以增强对干旱的抗性 ${ }^{[42]}$ 。所以中生植物可以更好的适应湿润生 境; 而旱中生植物对干旱的抗性更强。因此表现为不同水分生态类型植物叶脉密度对降水变化响应的差异。

4.3 不同叶脉类型植物叶片性状对降水变化的响应

增水使得矮生嵩草等平行叶脉植物的叶脉密度显著降低或不变,而使得花苜宿等羽状叶脉植物的叶脉密 度显著增加或不变; 与此相反, 减水使得垂穗披碱草等平行叶脉植物的叶脉密度显著增加或不变, 而使得花苜 葆等羽状叶脉植物叶脉密度显著减少或不变(图 2)。两类植物的响应存在明显的区别。叶脉投人的多少直 接决定着水分的输送能力 ${ }^{[47]}$ 。本研究结果表明平行叶脉植物的叶脉率是羽状叶脉植物的 4 倍(表 4)。叶脉 率的巨大差异可能是两类植物叶脉密度响应降水变化差异的原因。由于平行叶脉植物起源于中新世干旱环 境扩张的情景 ${ }^{[48]}$, 所以可能是平行叶脉植物的叶脉率较高的原因。这说明较高的叶脉投人是平行叶脉植物 应对干旱适应机制。所以在水分条件改善时,平行叶脉植物高的叶脉率使其无需继续提高叶脉密度就可以充 分利用水分; 甚至可能会因叶片增大而降低叶脉密度,因为叶脉的构建消耗大于叶肉 (分别为每克木质素和 纤维素 11.8 和 $6.5 \mathrm{mmol}$ 葡萄糖 ${ }^{[46]}$ 。由于叶脉率较低,生产力的提高, 增加了对传输组织的需求 ${ }^{[28,49]}$, 所以羽 状叶脉植物需要提高叶脉密度来提高水分的输送能力。

干旱通常导致更低的传输效率和出现栓塞的趋势, 要求植株在传输结构中的投资更高 ${ }^{[42]}$, 进而导致叶片 支撑结构投资的质量分数增加 ${ }^{[50]}$ 。当水分条件较差时, 平行叶脉植物提高叶脉密度, 保证水分的供给; 而羽 状叶脉植物只能通过相对保守的策略, 即通过降低叶脉密度减少叶脉的构建消耗。这表明平行叶脉植物较适 应干旱的生境, 而羽状叶脉植物更适应湿润的生境。

稳定碳同位素千分值是评估植物水分适应性的可靠途径 ${ }^{[51]}$ 。增水使得大部分羽状叶脉植物稳定碳同位 素千分值显著增加, 而减水使得大部分平行叶脉植物稳定碳同位素千分值显著减少。以往研究发现在全球尺 度上,稳定碳同位素千分值与年均降水存在显著的负相关性 ${ }^{[52]}$ 。小尺度 (夏威夷) 的研究表明,稳定碳同位 素千分值与年平均降水量呈显著负相关关系 ${ }^{[47]}$ 。对北美 Quercus suber 的研究也得到同样的结果 ${ }^{[53]}$ 。较低的 稳定碳同位素千分值使得 Quercus suber 在湿润的年份取得更多的地上生长量 ${ }^{\left[{ }^{[3]}\right.}$ 。这是因为水分适应性与土 壤中可利用水分和植物的生长状况密切相关 ${ }^{[54]}$ 。由于叶脉率的巨大差异, 平行叶脉植物较适应干旱的生境, 而羽状叶脉植物适应湿润的生境。所以在增水条件下, 羽状叶脉植物表现为有效适应湿润生境, 即增加稳定 碳同位素千分值; 同理在减水条件下,平行叶脉植物表现为有效适应干旱生境,即减少稳定碳同位素千分值。 这也进一步证明两类植物应对降水变化的模式不同。

4.4 叶片性状对增水和减水响应的敏感性

本实验中降水的变幅分别为 $\pm 50 \%$ 。从具体物种对两种模拟情形响应的差异来看,植物对于增水和减水 的响应不同(图 5)。部分植物性状对于增水具有显著响应,而对减水无显著响应; 反之亦然。同时,大部分植 物叶片性状对于增水和减水的敏感性不对称(表 3、图 5)。植物叶片性状对降水的敏感性不对称, 对增水的 
敏感性显著大于减水的敏感性 (图 5)。高寒草甸地区虽然年降水近 $600 \mathrm{~mm}$, 但是相对森林地区当地的降水 偏低。所以植物必然存在一系列的抗旱的机制 ${ }^{[6-7]}$ 。而当增水缓解干旱胁迫时, 植物的生长发育将得到很大 的促进 ${ }^{[5]}$ 。原有用于适应干旱的结构和功能并不能直接用于对增水的适应, 植物叶片对增水和减水的响应 不对称。所以未来降水增加可能促进当地植物群落的生长发育; 而周期波动中出现的干旱对植物影响较小。

\section{5 结论}

降水的变化对高寒草甸植物叶片性状具有显著的影响,但是不同性状的具体表现存在差异。植物叶片性 状在响应降水变化时,存在协同变化和相互制约。

不同水分生态类型的植物叶片性状对降水变化的响应相反,增水显著增加了中生植物叶片大小,降低其 叶脉密度,矮生嵩草的叶片大小增加了 $200.3 \%$, 叶脉密度减小了 $17.5 \%$, 而对旱中生植物的叶片大小和叶脉 密度的影响较小; 减水显著降低了旱中生植物叶片大小, 增加其叶脉密度,垂穗披碱草和异针茅的叶片大小分 别减少 $54.9 \%$ 和 $30.7 \%$, 其叶脉密度分别增加 $25 \%$ 和 $22.4 \%$, 而对中生植物的叶片大小和叶脉密度作用较小。

羽状叶脉植物表现为有效适应增水环境, 即增加叶脉密度和稳定碳同位素千分值,花苜宿、异叶米口袋的 叶脉密度的增加了 $7.8 \%$ 和 $4.0 \%$, 稳定碳同位素千分值增加 $2.5 \%$ 和 $3.3 \%$, 但是平行叶脉植物的叶脉密度和稳 定碳同位素千分值保持不变或降低; 而在减水条件下, 平行叶脉植物表现为有效适应干旱生境, 即增加叶脉密 度和降低稳定碳同位素千分值, 异针茅的叶脉密度增加了 $22.4 \%$, 稳定碳同位素千分值减小 $2.9 \%$, 而羽状叶 脉植物的叶脉密度不变或降低和稳定碳同位素千分值保持不变。

植物叶片性状对降水的敏感性不对称,对增水的敏感性显著大于减水的敏感性。植物在应对短期降水变 化时,植物形态可塑性的作用凸显,放大或缩小叶片大小是植物应对降水变化的最有效的途径,但是不同水分 生态类型和叶脉类型植物可塑性的方向存在显著差异。因此,降水变化可能改变不同植物的竞争能力,从而 改变群落组成,进而影响生态系统过程。

\section{参考文献 (References) :}

[ 1 ] Körner C. Through enhanced tree dynamics carbon dioxide enrichment may cause tropical forests to lose carbon. Philosophical Transactions of the Royal Society of London Series B-Biological Sciences, 2004, 359(1443) : 493-498.

[ 2 ] Schneider J V, Negraschis V, Habersetzer J, Rabenstein R, Wesenberg J, Wesche K, Zizka G. Taxonomic diversity masks leaf vein-climate relationships: lessons from herbarium collections across a latitudinal rainfall gradient in West Africa. Botany Letters, 2018, 165(3/4) : 384-395.

[ 3 ] Hudson J M G, Henry G H R, Cornwell W K. Taller and larger: shifts in Arctic tundra leaf traits after 16 years of experimental warming. Global Change Biology, 2011, 17(2) : 1013-1021.

[ 4 ] Ivanova L A, Yudina P K, Ronzhina D A, Ivanov L A, Hölzel N. Quantitative mesophyll parameters rather than whole - leaf traits predict response of $\mathrm{C}_{3}$ steppe plants to aridity. New Phytologist, 2018, 217(2): 558-570.

[ 5 ] Dong N, Prentice I C, Wright I J, Evans B J, Togashi H F, Caddy - Retalic S, McInerney F A, Sparrow B, Leitch E, Lowe A J. Components of leaf-trait variation along environmental gradients. New Phytologist, 2020, 228(1) : 82-94.

[ 6 ] Meehl G A, Stocker T F, Collins W D, Friedlingstein P, Gaye A T, Gregory J M, Kitoh A, Knutti R, Murphy J M, Noda A, Raper S C B, Watterson I G, Weaver A J, Zhao Z C. Global climate projections//Solomon S, ed. Climate Change 2007: The Physical Science Basis. Contribution of Working Group I to the Fourth Assessment Report of the Intergovernmental Panel on Climate Change. New York: Cambridge University Press, 2007: 747-845

[ 7 ] Xu X K, Chen H, Levy J K. Spatiotemporal vegetation cover variations in the Qinghai-Tibet Plateau under global climate change. Chinese Science Bulletin, 2008, 53(6): 915-922.

[ 8 ] Wang S P, Duan J C, Xu G P, Wang Y F, Zhang Z H, Rui Y C, Luo C Y, Xu B, Zhu X X, Chang X F, Cui X Y, Niu H S, Zhao X Q, Wang W Y. Effects of warming and grazing on soil N availability, species composition, and ANPP in an alpine meadow. Ecology, 2012, 93 (11) : 2365-2376

[ 9 ] Wang S P, Meng F D, Duan J C, Wang Y F, Cui X Y, Piao S L, Niu H S, Xu G P, Luo C Y, Zhang Z H, Zhu X X, Shen M G, Li Y N, Du M Y, Tang Y H, Zhao X Q, Ciais P, Kimball B, Peñuelas J, Janssens I A, Cui S J, Zhao L, Zhang F W. Asymmetric sensitivity of first flowering date to warming and cooling in alpine plants. Ecology, 2014, 95(12) : 3387-3398. 
[10] Piao S L, Fang J Y, He J S. Variations in vegetation net primary production in the Qinghai-Xizang Plateau, China, from 1982 to 1999. Climatic Change, 2006, 74(1/3): 253-267.

[11] Wang S P, Yang X X, Lin X W, Hu Y G, Luo C Y, Xu G P, Zhang Z H, Su A L, Chang X F, Chao Z G, Duan J C. Methane emission by plant communities in an alpine meadow on the Qinghai-Tibetan Plateau: a new experimental study of alpine meadows and oat pasture. Biology Letters, $2009,5(4): 535-538$.

[12] Cornwell W K, Ackerly D D. Community assembly and shifts in plant trait distributions across an environmental gradient in coastal California. Ecological Monographs, 2009, 79(1): 109-126.

[13] Jung V, Albert C H, Violle C, Kunstler G, Loucougaray G, Spiegelberger T. Intraspecific trait variability mediates the response of subalpine grassland communities to extreme drought events. Journal of Ecology, 2014, 102(1) : 45-53.

[14] Sandel B, Goldstein L J, Kraft N J B, Okie J G, Shuldman M I, Ackerly D D, Cleland E E, Suding K N. Contrasting trait responses in plant communities to experimental and geographic variation in precipitation. New Phytologist, 2010, 188(2) : 565-575.

[15] 中国科学院中国植物志编辑委员会. 中国植物志. 北京: 科学出版社, 2004: 35-36.

[16] Ungar I A. Are biotic factors significant in influencing the distribution of halophytes in saline habitats? The Botanical Review, 1998, 64 (2) : 176- 199.

[17] 中国科学院植物研究所, 中国科学院长春地理研究所. 西藏植被. 北京: 科学出版社, 1988: 66-76.

[18] Szabados L, Kovács H, Zilberstein A, Bouchereau A. Plants in extreme environments: Importance of protective compounds in stress tolerance. Advances in Botanical Research, 2011, 57: 105-150.

[19] Csipkés M. The examination of the profitability and competitiveness regarding the energy plantations of woody plants in the region of Észak-Alföld. Applied Studies in Agribusiness and Commerce, 2012, 6(5): 5-11.

[20] Luo C Y, Xu G P, Chao Z G, Wang S P, Lin X W, Hu Y G, Zhang Z H, Duan J C, Chang X F, Su A L, Li Y N, Zhao X Q, Du M Y, Tang Y H, Kimball B. Effect of warming and grazing on litter mass loss and temperature sensitivity of litter and dung mass loss on the Tibetan plateau. Global Change Biology, 2010, 16(5): 1606-1617.

[21] Nardini A, Pedà G, La Rocca N. Trade-offs between leaf hydraulic capacity and drought vulnerability : morpho-anatomical bases, carbon costs and ecological consequences. New Phytologist, 2012, 196(3) : 788-798.

[22] Sack L, Scoffoni C. Leaf venation: structure, function, development, evolution, ecology and applications in the past, present and future. New Phytologist, 2013, 198(4): 983-1000.

[23] Muhaidat R, Sage R F, Dengler N G. Diversity of Kranz anatomy and biochemistry in $\mathrm{C}_{4}$ eudicots. American Journal of Botany, 2007, 94( 3) : 362-381.

[24] McKown A D, Dengler N G. Vein patterning and evolution in $\mathrm{C}_{4}$ plants. Botany, 2010, 88(9) : 775-786.

[25] Wang C S, Lyu W W, Jiang L L, Wang S P, Wang Q, Meng F D, Zhang L R. Changes in leaf vein traits among vein types of alpine grassland plants on the Tibetan Plateau. Journal of Mountain Science, 2020, 17(9): 2161-2167.

[26] Scoffoni C, Rawls M, McKown A, Cochard H, Sack L. Decline of leaf hydraulic conductance with dehydration: relationship to leaf size and venation architecture. Plant Physiology, 2011, 156(2) : 832-843.

[27] 杨晓霞, 任飞, 周华坤, 贺金生. 青藏高原高寒草甸植物群落生物量对氮、磷添加的响应. 植物生态学报, 2014, 38(2): 159-166.

[28] Sack L, Scoffoni C, McKown A D, Frole K, Rawls M, Havran J C, Tran H, Tran T. Developmentally based scaling of leaf venation architecture explains global ecological patterns. Nature Communications, 2012, 3: 837.

[29] Ueno O, Kawano Y, Wakayama M, Takeda T. Leaf vascular systems in $\mathrm{C}_{3}$ and $\mathrm{C}_{4}$ grasses : a two-dimensional analysis. Annals of Botany, 2006, 97 (4): 611-621.

[30] Luo T X, Li M C, Luo J. Seasonal variations in leafo ${ }^{13} \mathrm{C}$ and nitrogen associated with foliage turnover and carbon gain for a wet subalpine fir forest in the Gongga Mountains, eastern Tibetan Plateau. Ecological Research, 2011, 26(2) : 253-263.

[31] Niklas K J, Cobb E D, Niinemets Ü, Reich P B, Sellin A, Shipley B, Wright I J. "Diminishing returns" in the scaling of functional leaf traits across and within species groups. Proceedings of the National Academy of Sciences of the United States of America, 2007, 104(21): 8891-8896.

[32] 冯秋红, 程瑞梅, 史作民, 刘世荣, 刘兴良, 何飞, 曹慧明. 巴郎山刺叶高山栋叶片 $\delta^{13} \mathrm{C}$ 对海拔高度的响应. 生态学报, 2011, 31 (13): 3629-3637.

[33] Schmid B. The species richness-productivity controversy. Trends in Ecology \& Evolution, 2002, 17(3): 113-114.

[34] Milla R, Reich P B. The scaling of leaf area and mass: the cost of light interception increases with leaf size. Proceedings of the Royal Society BBiological Sciences, 2007, 274(1622): 2109-2114.

[35] Peppe D J, Royer D L, Cariglino B, Oliver S Y, Newman S, Leight E, Enikolopov G, Fernandez-Burgos M, Herrera F, Adams J M, Correa E, Currano E D, Erickson J M, Hinojosa L F, Hoganson J W, Iglesias A, Jaramillo C A, Johnson K R, Jordan G J, Kraft N J B, Lovelock E C, 
Lusk C H, Niinemets Ü, Peñuelas J, Rapson G, Wing S L, Wright I J. Sensitivity of leaf size and shape to climate: global patterns and paleoclimatic applications. New Phytologist, 2011, 190(3) : 724-739.

[36] Reich P B, Oleksyn J. Climate warming will reduce growth and survival of Scots pine except in the far north. Ecology Letters, 2008, 11(6) : 588-597.

[37] Wright I J, Westoby M. Leaves at low versus high rainfall: coordination of structure, lifespan and physiology. New Phytologist, 2002, 155(3): 403-416.

[38] Noda H, Muraoka H, Washitani I. Morphological and physiological acclimation responses to contrasting light and water regimes in Primula sieboldii. Ecological Research, 2004, 19(3): 331-340.

[39] Fonseca C R, Overton J M, Collins B, Westoby M. Shifts in trait-combinations along rainfall and phosphorus gradients. Journal of Ecology, 2000, 88(6) : 964-977.

[40] Yates M J, Verboom G A, Rebelo A G, Cramer M D. Ecophysiological significance of leaf size variation in proteaceae from the cape floristic region. Functional Ecology, 2010, 24(3): 485-492.

[41] Yang Y, Wang G X, Klanderud K, Yang L D. Responses in leaf functional traits and resource allocation of a dominant alpine sedge (Kobresia pygmaea) to climate warming in the Qinghai-Tibetan Plateau permafrost region. Plant And Soil, 2011, 349(1/2) : 377-387.

[42] Westoby M, Wright I J. The leaf size-twig size spectrum and its relationship to other important spectra of variation among species. Oecologia, 2003, $135(4): 621-628$.

[43 ] Wright I J, Reich P B, Westoby M, Ackerly D D, Baruch Z, Bongers F, Cavender-Bares J, Chapin T, Cornelissen J H C, Diemer M, Flexas J, Garnier E, Groom P K, Gulias J, Hikosaka K, Lamont B B, Lee T, Lee W, Lusk C, Midgley J J, Navas M L, Niinemets Ü, Oleksyn J, Osada N, Poorter H, Poot P, Prior L, Pyankov V I, Roumet C, Thomas S C, Tjoelker M G, Veneklaas E J, Villar R. The worldwide leaf economics spectrum. Nature, 2004, 428(6985): 821-827.

[44] Golodets C, Sternberg M, Kigel J. A community-level test of the leaf-height-seed ecology strategy scheme in relation to grazing conditions. Journal of Vegetation Science, 2009, 20(3): 392-402.

[45] Sultan S E. An emerging focus on plant ecological development. New Phytologist, 2005, 166(1) : 1-5.

[46] Lambers H, Poorter H. Inherent variation in growth rate between higher plants: a search for physiological causes and ecological consequences. Advances in Ecological Research, 2004, 34: 283-362.

[47] Dunbar-Co S, Sporck M J, Sack L. Leaf trait diversification and design in seven rare taxa of the Hawaiian plantago radiation. International Journal of Plant Sciences, 2009, 170(1): 61-75.

[48] Edwards E J, Osborne C P, Strömberg C A E, Smith S A, C 4 Grasses Consortium. The origins of $\mathrm{C}_{4}$ grasslands: integrating evolutionary and ecosystem science. Science, 2010, 328(5978) : 587-591.

[49] Brodribb T J, Holbrook N M. Forced depression of leaf hydraulic conductance in situ: effects on the leaf gas exchange of forest trees. Functional Ecology, 2007, 21(4) : 705-712.

５0］何士敏, 汪建华, 秦家顺. 几种沙棘叶片组织结构特点和抗旱性比较. 林业科技开发, 2009, 23(1): 16- 19.

[51] Von Caemmerer S, Ludwig M, Millgate A, Farquhar G D, Price D, Badger M, Furbank R T. Carbon isotope discrimination during $\mathrm{C}_{4}$ photosynthesis: insights from transgenic plants. Australian Journal of Plant Physiology, 1997, 24(4) : 487-494.

[52] Diefendorf A F, Mueller K E, Wing S L, Koch P L, Freeman K H. Global patterns in leaf ${ }^{13} \mathrm{C}$ discrimination and implications for studies of past and future climate. Proceedings of the National Academy of Sciences of the United States of America, 2010, 107(13) : 5738-5743.

[53] Ramírez-Valiente J A, Sánchez-Gómez D, Aranda I, Valladares F. Phenotypic plasticity and local adaptation in leaf ecophysiological traits of 13 contrasting cork oak populations under different water availabilities. Tree Physiology, 2010, 30(5) : 618-627.

[54] Luo C Y, Xu G P, Wang Y F, Wang S P, Lin X W, Hu Y G, Zhang Z H, Chang X F, Duan J C, Su A L, Zhao X Q. Effects of grazing and experimental warming on DOC concentrations in the soil solution on the Qinghai-Tibet plateau. Soil Biology and Biochemistry, 2009, 41(12): 2493-2500. 